\title{
Expression of Cytokeratin 7 and 20 in Pathological Conditions of the Bile Tract
}

\author{
Daniela Cabibi ${ }^{1}$, Anna Licata², Elisa Barresi ${ }^{1}$, Antonio Craxi², \\ Federico Aragona ${ }^{1}$ \\ 1 Department of Pathology, \\ ${ }^{2}$ Gastroenterology and Hepatology Unit, Department of Internal Medicine, University of \\ Palermo, Italy
}

\section{Summary}

Expression of cytokeratin 7 (CK7) and cytokeratin 20 (CK20) helps to establish the origin of biliary and metastatic carcinomas. We investigated the expression of CK7 and CK20 in inflammatory, metaplastic and neoplastic conditions of the bile ducts, and evaluated possible relationships between the CK expression pattern and extrahepatic bile duct/gallbladder carcinomas (EBDCs) or intrahepatic bile duct carcinomas (IBDCs).

We used immunohistochemistry for the investigation of 48 formalin-fixed, paraffin-embedded specimens grouped as: A) lithiasic or inflamed surgically resected extrahepatic bile ducts/gallbladders: all were $\mathrm{CK} 7^{+} / \mathrm{CK} 20^{+}$; B) percutaneous liver biopsies from patients with chronic hepatitis $\mathrm{C}$ primary biliary cirrhosis and primary sclerosing cholangitis: all were $\mathrm{CK} 7^{+} / \mathrm{CK} 20^{-}$; C) EBDCs: all were $\mathrm{CK} 7^{+} / \mathrm{CK} 20^{+}$, except for two cases which were CK7-/CK20-; D) IBDCs: all were $\mathrm{CK} 7^{+} / \mathrm{CK} 20^{-}$, except for one case showing CK20 positivity. Metaplastic changes were seen only among specimens in groups A and C: in these cases, CK20 was either focally or diffusely expressed.

Our study suggests that the expression of cytokeratins under specific stimuli can be different from normal tissues, and that sometimes CK20 expression can be related to and precede the occurrence of metaplastic alterations.

Key words: Cytokeratin 7 (CK7) - Cytokeratin 20 (CK20) - Bile duct tumors - Intestinal metaplasia

\section{Introduction}

For many years, the possibility of a causal relationship between lithiasis and carcinoma of the biliary tract has been under debate. In the gallbladder and extrahepatic bile tract, chronic inflammation has been accepted as a factor participating in the multistep process leading to cancer $[10,31]$. It has also been associated with the presence of gastric and intestinal metaplasia and with pre-cancerous lesions $[1-3,5-7,13,15,17]$. By contrast, in intrahepatic bile duct carcinoma, the role of stones and inflammation is still controversial because hepatolithiasis is rare in Western countries, and more than $80 \%$ of cholangiocarcinomas have no history of predisposing factors [21].

The expression of cytokeratins 7 (CK7) and cytokeratin 20 (CK20) in the biliary system has been assessed in a few studies, and the results are confusing $[8,26,27]$. Moll found that neoplasms usually repeat the same CK7 and CK20 expression pattern of the epithelia from which they originate [19]. Recently, Faa reported that CK20 is a "bile duct type" cytokeratin, and that its expression differs in experimental models of bile duct and oval cell proliferation, suggesting the existence of different mech-

Address for correspondence: Anna Licata, Gastroenterology and Hepatology Unit, Department of Internal Medicine, University of Palermo, Piazza delle Cliniche n. 2, 90127 Palermo, Italy. Phone: +39 091655 2280, Fax: +390916552156.

E-mail: annalisalicata@yahoo.com 
anisms regulating proliferation and differentiation of biliary epithelial cells [9]. Molecular studies of cholangiocarcinogenesis in rat livers have shown that alterations induced by furan treatment (intestinal metaplasia and "type intestinal" of cholangiocarcinoma) are different from hyperplastic bile ductular alterations induced by common bile duct ligation [24]. Hence, different pathogenetic conditions appear to be responsible for the occurrence of bile duct carcinomas, and the correlation between inflammatory (lithiasis) or proliferative stimuli (ductular proliferation) and metaplastic alterations is still unclear.

In an attempt to clarify the relationship between metaplastic phenomena and the chain of events leading to bile duct cancer, we used immunohistochemical methods for the investigation of the expression pattern of CK7 and CK20 in the gallbladder and in the intraand extrahepatic bile ducts, aiming to correlate the expression pattern of cytokeratins with conditions which usually precede the occurrence of bile duct neoplasia. Since an ultrastructural, histochemical and immunohistochemical similarity between the gallbladder and the extrahepatic bile ducts both in normal and in neoplastic conditions is commonly accepted by various investigators [26, 27], we pooled together gallbladder and extrahepatic bile duct malignancy as "Extrahepatic Bile Duct Carcinoma" (EBDC), which is morphologically different from "Intrahepatic Bile Duct Carcinoma" (IBDC), whose features are still ambiguous [8, 26].

\section{Materials and Methods}

\section{Specimens}

Between March 1998 and December 2001, we selected our cases by a systematic search through the pathology reports of the Department of Pathology of the University of Palermo. lows:

We identified 48 specimens that were grouped as fol-

A) Inflamed gallbladders and extrahepatic bile ducts: 10 surgical specimens of lithiasic and/or inflamed gallbladders and extrahepatic bile ducts;

B) 15 percutaneous liver biopsy specimens obtained from patients with chronic hepatitis $\mathrm{C}$, with primary biliary cirrhosis or primary sclerosing cholangitis;

C) EBDCs: 13 surgically resected specimens, 10 from gallbladder and 3 from large bile ducts;
D) IBDCs: 10 specimens, 5 obtained by surgical resection and 5 by percutaneous liver biopsy. Two of these cases were associated with sclerosing cholangitis, and one with intrahepatic bile duct stones.

\section{Immunohistochemistry}

Formalin-fixed, paraffin-embedded tissue sections were stained by Hematoxylin-Eosin and Alcian blue-PAS. Immunohistochemical studies were done using the Avidin-Biotin-Complex (ABC) technique. The primary monoclonal antibodies (mAbs) used were CK7 (clone OV-TL12/30) and CK20 (clone Ks 20.8), both obtained from DAKO (A/S, Glostrup, Denmark).

Sections were cut and then deparaffinized in xylene and rehydrated through alcohols. To improve immunostaining, samples were digested with $0.1 \%$ trypsin before incubation with $\mathrm{CK} 7 \mathrm{mAb}$, and microwaved in 10 mM citrate buffer ( $\mathrm{pH}$ 6.0) before incubation with CK20 mAb. After incubation with CK7 and CK20 mAbs for 25 minutes at room temperature, sections were incubated with the secondary antibody, which was followed by $\mathrm{ABC}$, as described in the manufacturer's instructions of Universal LSAB (Dako). Amino-Ethil-Carbazole (AEC) was used as chromogen. The slides were then counterstained with hematoxylin and routinely mounted. As a positive control for CK7, we used intrahepatic bile ducts present in each section; for CK20, immunostaining was carried out on a section of rectal adenocarcinoma. For both $\mathrm{mABs}$, hepatocytes served as a negative control.

\section{Quality assessment}

Each section was analyzed by two independent observers. As previously shown by Moll [19], in non-inflamed, non-lithiasic bile system, there was a strong and diffuse immunoreactivity against CK7 mAb, whereas less than $1 \%$ of cells were reactive against CK20 mAb (Fig. 1A-B). Therefore, immunostaining for CK7 and CK20 was considered negative when less than $1 \%$ of cells were positively stained in the whole section.

\section{Results}

Results are summarized in Table 1.

Group A: Inflamed gallbladders and extrahepatic bile ducts showed focal or diffuse hyperplastic, metaplastic and sometimes dysplastic phenomena. All of them were CK $7^{+} / \mathrm{CK} 20^{+}$(Fig. 1C-E).

Fig. 1. A-B: Normal gallbladder strongly reactive for CK7 $(\mathrm{A}, \times 100)$ and negative for CK20 $(\mathrm{B}, \times 100)$.

C-D: Inflamed gallbladder showed focal $(C, \times 100)$ or diffuse positivity for CK20 in metaplastic and hyperplastic areas $(D \times 250)$.

E: Hyperplastic area showed positivity for CK20 $(\times 250)$; F: Metaplastic and focally dysplastic area immunoreactive for CK20 $(\times 250)$. G-H: CK20 expression alone in surgically resected EBDC $(\mathrm{G}, \times 100)$ and CK7 alone in a percutaneous liver biopsy of IBDC (H, $\times 100)$. 
Cytokeratin 7 and 20 in Bile Tract · 67
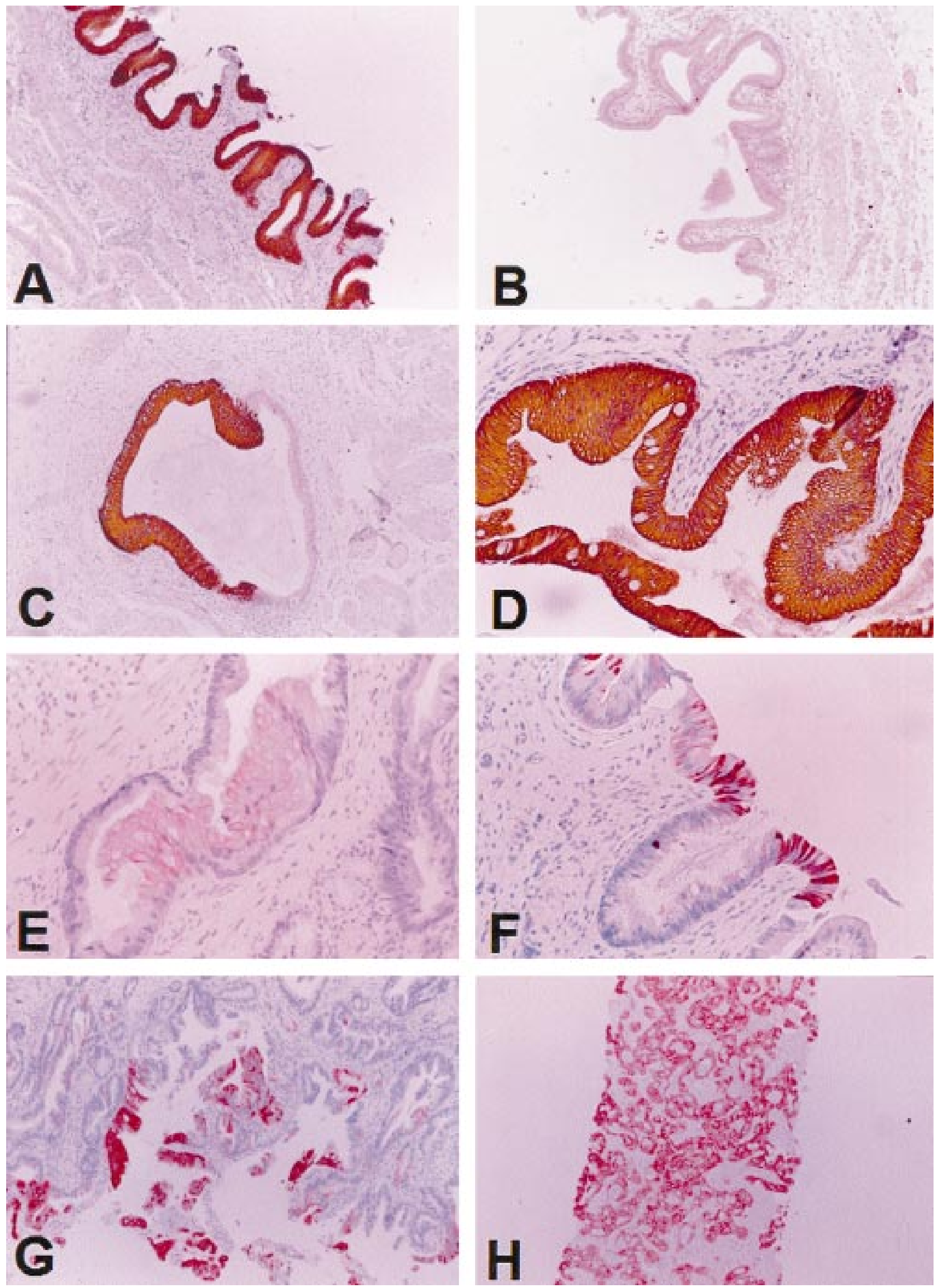
Table 1. CK 7 and 20 expression in normal tissues, inflamed intra and extrahepatic bile ducts, EBDCs and EBDCs.

\begin{tabular}{lll}
\hline & CK 7 & CK20 \\
\hline Normal Tissues & + & - \\
Extrahepatic Bile Ducts & + & $+/ *$ \\
Intrahepatic Bile Ducts & + & - \\
Extrahepatic Bile Duct Carcinomas & + & + \\
Intrahepatic Bile Duct Carcinomas & + & $-* *$ \\
\hline
\end{tabular}

* Focal staining pattern for CK20 in inflamed extrahepatic bile ducts was evident in areas of metaplastic phenomena (intestinal or foveolar).

** Only one case was CK20 positive among IBDCs, the "hepatolithiasis associate case".

Group B: Inflamed intrahepatic bile ducts of liver biopsies were always $\mathrm{CK} 7^{+} / \mathrm{CK} 20^{-}$. In these specimens, metaplastic changes were never expressed.

Group C: Gallbladder carcinomas and EBDCs were mostly CK $7^{+} / \mathrm{CK} 20^{+}$. CK20 showed either a focal or a diffuse staining pattern, which was also present in the adjacent dysplastic and/or metaplastic mucosa (Fig. $1 \mathrm{~F}-\mathrm{G})$. Two cases of this group were CK7-/CK20-. Morphologically, these were mucinous carcinomas with a signet ring cell component. Goblet cell metaplasia was never present in the adjacent mucosa, but there were many areas of diffuse pseudopyloric metaplasia, which were negative for CK7 and CK20.

Group D: IBDCs were CK7+/CK20- (Fig 1H). Only one specimen showed focal positivity for CK20. This patient had intrahepatic bile duct stones.

In general, the expression of CK20 in the extrahepatic bile system was always associated with goblet cell and/or foveolar metaplastic alterations. The metaplastic surface epithelium was positive for CK20 (Fig. 1D), even if sometimes apparently non-metaplastic cells were also CK20 positive (Fig. 1C). Intensity and spread of the CK20 expression were independent from the grade of differentiation of EBDCs. Conversely, IBDC cases did not show CK20, even independently of the grade of differentiation, except for the patient with intrahepatic bile duct stones.

\section{Discussion}

In our study, we showed that in inflamed gallbladder and extrahepatic bile ducts of group A, CK7 is always expressed as in normal tissues [19] whereas CK20 is also expressed and related to the presence of hyperplastic and metaplastic phenomena (goblet cell and/or pseudopyloric surface metaplasia). In areas of gastric metaplasia, surface foveolar metaplastic epithelia often express both CK7 and CK20. By contrast, metaplastic pseudopyloric glands are often negative for both, a finding similar to that reported by Moll and Remakaers for normal gastric epithelium [19, 25]. Small normal and proliferating bile ducts of group B are always $\mathrm{CK} 7^{+} / \mathrm{CK} 20^{-}$as usually described for ductular structures; metaplastic changes were never expressed.

Extrahepatic bile duct carcinomas are $\mathrm{CK} 7^{+} / \mathrm{CK} 20^{+}$ and often associated with metaplastic phenomena and expression of CK20 in the adjacent mucosa. We did not confirm the data reported by Duval et al. that the majority of EBDCs are $\mathrm{CK} 7^{+} / \mathrm{CK} 20^{-}$, which is probably due to the use of a different cut-off level for positivity to assess CK20 immunostaining [8].

All intrahepatic bile duct carcinomas were $\mathrm{CK} 7^{+} / \mathrm{CK} 20^{-}$. They never showed metaplastic changes or CK20 expression, neither in neoplastic areas nor in adjacent non neoplastic bile ducts, except for the case of the patient with intrahepatic bile duct stones, which showed focal positivity for CK20.

Regarding these data, our results are in contrast to those of Rullier [26], who reported that about $47 \%$ of peripheral cholangiocarcinomas were positive for CK20, and to the results of Shimonishi [27], who showed scattered positivity for CK20 in moderately and poorly differentiated IBDCs. We found that the large majority of IBDCs did not express CK20, and that the expression pattern of cytokeratins was independent from the differentiation grade. In general, we agree with Rullier, who demonstrated a variability of CK20 positivity according to the different location of tumors in the biliary tract, with an increase in expression ranging from low in IBDCs (called "peripheral carcinomas") to high in EBDCs ("non peripheral carcinomas"). We found only one case of IBDCs in association with intrahepatic bile duct stones (or hepatolithiasis), which was diffusely positive for CK7 and focally positive for CK20. This cytokeratin expression pattern was similar to that of EBDCs. As hepatolithiasis is rare in Western countries, we did not find any other case of intrahepatic bile duct stones. Previous studies demonstrated that in livers with hepatolithiasis, intrahepatic bile ducts have metaplastic changes similar to the well-known metaplastic lesions in the gallbladder [14]. These cases sometimes evolve in cholangiocarcinoma $[12,22,23,29,30]$. We feel that hepatolithiasis may be the underlying condition related to the appearance of CK20 expression in IBDCs.

Metaplastic phenomena suggest the pluripotentiality of cells during the differentiation process and the liability to determine malignant transformation [13]. In our study, it was evident that CK20 was not only expressed in intestinal metaplastic goblet cells epithelia, but also appeared in hyperplastic or even in morphologically normal epithelia, suggesting that cytokeratin expression may precede morphologic alterations. Thus, CK20 expression in an inflamed bile system could be an "early marker" of metaplasia. About $80 \%$ of our EBDCs are 
$\mathrm{CK} 7^{+} / \mathrm{CK} 20^{+}$; hence, they probably derive from goblet cells and/or pseudopyloric surface metaplasia, which is detected by CK20 mAb [19]. We found that two EBDCs cases were negatively stained for both cytokeratins, while in the adjacent mucosa, there was no goblet cells metaplasia, but only pseudopyloric gland metaplasia. Although the role of pseudopyloric metaplasia in the sequence metaplasia $\rightarrow$ dysplasia $\rightarrow$ carcinoma is still controversial [28], we think that cancer can arise from metaplastic pseudopyloric glands which are CK7-/CK20-, like their normal counterpart in the stomach [19].

Rullier recently hypothesized that CK20 expression in peripheral and non-peripheral cholangiocarcinomas is determined by the embryology of the biliary tree [26]. Instead, we suggest the hypothesis that these tumors are embriologically similar, but their peculiar cytokeratin pattern depends on a diverse pathogenetic sequence underscoring two mechanisms of carcinogenesis. Concerning non-peripheral cholangiocarcinomas, under a chronic inflammatory stimulus, the most likely sequence is metaplasia $\rightarrow$ dysplasia $\rightarrow$ cancer. The appearance of CK20 expression could be its hallmark. Conversely, the expression pattern of our peripheral cholangiocarcinomas was $\mathrm{CK} 7^{+} / \mathrm{CK} 20^{-}$, like that of normal and proliferating non neoplastic intrahepatic bile ducts. Therefore, under a chronic proliferative stimulus, a different sequence bypassing the "metaplasia step" could be the starter of most Western peripheral cholangiocarcinomas through the following sequence: ductular proliferation $\rightarrow$ dysplasia $\rightarrow$ cancer. Our hypothesis, based on the ability of biliary cells to express both cytokeratins, is supported by experimental observations in a model of bile duct and oval cell proliferation [9] and by molecular studies of rat liver cholangiocarcinogenesis [24].

In conclusion, our study shows that the expression of CK7 and 20, under specific stimuli, can be different from normal tissues and related to the appearance of metaplasia. We suggest that CK7 and 20 expression patterns can be useful for identifying metaplastic alterations at the onset of the biliary tumors, and thus have diagnostic usefulness. Assessments of larger series of Western and Eastern patients will test the concept that "peripheral" and "non-peripheral" cholangiocarcinomas are immunologically different, not because of embriologic reasons, but as a result of a different multistep pathogenesis.

\section{References}

1. Albores-Saavedra J, Alcantra-Vasquez A, Cruz-Oritz H, Herrera-Goepfert R (1980) The precursor lesions of invasive gallbladder carcinoma: Hyperplasia, atypical hyperplasia and carcinoma in situ. Cancer 45: 919-927

2. Albores-Saavedra J, Henson DE (1986) Tumors of the gallbladder and extrahepatic bile ducts. In: Atlas of Tumor
Pathology, $2^{\text {nd }}$ series, fascicle 22. Washington DC: Armed Forces Institute of Pathology

3. Albores-Saavedra J, Nadji M, Henson DE (1986) Intestinal type adenocarcinoma of the gallbladder: a clinicopathologic and immunohistochemical study of seven cases. Am J Surg Pathol 10: 19-25

4. Albores-Saavedra J, Nadj M, Henson DE, Ziegels-Weissman J, Mones JM (1986) Intestinal metaplasia of the gallbladder: a morphologic and imunohistochemical study. Hum Pathol 17: 614-620

5. Azadeh B, Parai SK (1980) Argentaffin cells, intestinal metaplasia and antral metaplasia in carcinoma of the gallbladder. Histopathology 4: 653-659

6. Dowling GP, Kelly JK (1986) The histogenesis of adenocarcinoma of the gallbladder. Cancer 58: 1702-1708

7. Duarte I, Llanos O, Domke H, Harz C, Valdivieso V (1993) Metaplasia and precursor lesions of gallbladder carcinoma. Cancer 72: 1878-1884

8. Duval JV, Savas L, Banner BF (2000) Expression of cytokeratins 7 and 20 in carcinomas of the extrahepatic biliary tract, pancreas and gallbladder. Arch Pathol Lab Med 124 (8): 1196-1200

9. Faa G, Van Eyken P, Roskams T, Miyazaki H, Serreli S, Ambu R (1998) Expression of cytokeratin 20 in developing rat liver and in experimental models of ductular and oval cell proliferation. J Hepatol 29: 628-33

10. Hart J, Shani M, Modan B (1972) Epidemiological aspects of gallbladder and biliary tract neoplasm. Am J Public Heath 62: 36-42

11. Kijima H, Watanabe H, Iwafuchi M, Ishihara N (1989) Histogenesis of gallbladder carcinoma from investigation of early carcinoma and microcarcinoma. Acta Pathol JPN 39: 235-244

12. Koga A, Ichinomiyia H, Yamaguchi K, Miyazaki K, Nakayama F (1985) Hepatolithiasis associated with cholangiocarcinoma. Cancer 55: 2826-2829

13. Kozuka S, Kurashina M, Tsubone M, Hachisuka K, Yasui A (1984) Significance of intestinal metaplasia for the evolution of cancer in the biliary tract. Cancer 54: 2277-2285

14. Kurumaya H, Terada T, Nakanuma Y (1990) "Metaplastic lesions" in intrahepatic bile ducts in hepatolithiasis. An histochemical and immunohistochemical study. J Gastroenterol Hepatol 5: 530-536

15. Laitio M, Hakkinen I (1975) Intestinal-type carcinoma of gallbladder: a histochemical and immunological study. Cancer 36: 1668-1674

16. Laitio M (1983) Histogenesis of epithelial neoplasms of the gallbladder. I. Dysplasia. Pathol Res Pract 178: 51-56

17. Laitio M (1983) Histogenesis of epithelial neoplasms of human gallbladder: II. Classification of carcinoma on the basis of morphological features. Pathol Res Pract 178: 57-66

18. Laitio M (1983) Carcinoma of the extrahepatic bile ducts: a histopathologic study. Pathol Res Pract 178: 67-72

19. Moll R, Lowe A, Laufer J, Franke WW (1992) Cytokeratin 20 in human carcinomas. Am J Pathol 140: 427-447

20. Nakajo S, Yamamoto M, Tahara E (1990) Morphometrical analysis of gallbladder adenoma and adenocarcinoma with reference to histogenesis and adenoma-carcinoma sequences. Virchows Arch A Pathol Anat Histopathol 417: 49-56 
70 . D. Cabibi et al.

21. Nakanuma Y, Hoso M, Terada T (1997) Clinical and pathological features of cholangiocarcinoma. In: Okuda K, Tabor E, (Eds.) Liver Cancer, pp 279-290. Churchill Livingstone, New York

22. Nakanuma Y, Terada T, Tanaka Y, Ohta G (1985) Are hepatolithiasis and cholangiocarcinoma aetiologically related? A morphological study of 12 cases of hepatolithiasis associated with cholangiocarcinoma. Virchow Arch 406: $45-58$

23. Ohta T. Nagakawa T, Konishi I (1988) Clinical experience of intrahepatic cholangiocarcinoma associated with hepatolithiasis. Japanese J Surg 18: 47-53

24. Radaeva S, Ferreira Gonzalez A, Sirica AE (1999) Overexpression of C-neu and C-met during rat liver cholangiocarcinogenesis: A link between biliary intestinal metaplasia and mucin-producing cholangiocarcinoma. Hepatology 29: 1453-1462

25. Ramaekers F, Van Niekerk C, Poels L, Schaafsma E, Huijsmans A, Robben H, Schaart G, Vooijs P (1990) Use of monoclonal antibodies to keratin 7 in the differential diagnosis of adenocarcinomas. Am J Pathol 136: 641-655

26. Rullier A, Le Bail B, Fawaz R, Blank JF, Saric J, BioulacSage P (1998) Cytokeratin 7 and 20 expression in cholangiocarcinomas varies along the biliary tract but still differs from that in colorectal carcinoma metastasis. Am J Surg Pathol 24 (6): 870-876

27. Shimonishi T, Miyazaki K, Nakanuma Y (2000) Cytokeratin profile relates to histological subtypes and intra location of intrahepatic cholangiocarcinoma and primary sites of metastatic adenocarcinoma of liver. Histopatology 37 (1): 55-63

28. Sternberg S.S. (1999) Diagnostic Surgical Pathology, 3th edn, pp 1647-1648. Lippincott, Williams and Wilkins, Philadelphia

29. Terada T, Nakanuma Y, Ohta T, Nagakawa T (1992) Histological features and interphase nucleolar organizer regions in hyperplastic, dysplastic and neoplastic epithelium of intrahepatic bile ducts in hepatolithiasis. Histopathology $21: 233-240$

30. Terada T, Kurumaya H, Nakanuma Y(1989) Intrahepatic cholesterol stones associated with peripheral intrahepatic cholangiocellular carcinoma: An autopsy case. Am. J Gastroenterol 84: 1431-1433

31. Warren R, Balch FG (1940) Carcinoma of the gallbladder: The etiological role of gallstones. Surgery: 657-662

Received: October 30, 2002

Accepted in revised version: February 21, 2003 